\title{
Self-tracking as communication
}

Lomborg, Stine; Frandsen, Kirsten

Published in:

Information, Communication \& Society

DOI:

10.1080/1369118X.2015.1067710

Publication date:

2016

Document version

Peer reviewed version

Citation for published version (APA):

Lomborg, S., \& Frandsen, K. (2016). Self-tracking as communication. Information, Communication \& Society, 19(7), 1015-1027. https://doi.org/10.1080/1369118X.2015.1067710 
Lomborg, S. \& Frandsen, K. (2016). Self-tracking as communication. [pre-print version]

Citation: Lomborg, S. \& Frandsen, K. (2016). Self-tracking as communication. Information, Communication and Society, 19(7): 1015-1027.

\section{Self-tracking as communication}

\section{Introduction}

In the abundant market of digital mobile technologies and services, self-tracking services, including dedicated smartphone applications and wearable technologies such as smart watches, wristbands and glasses seems to constitute the 'new hype'. These technologies allow ordinary individuals to systematically monitor, document and analyze an array of aspects of daily life, including sleep patterns, mood, calorie intake, heart rate and physical exercise. Allegedly, self-tracking grants the individual user more and deeper self-knowledge (Wolf, 2010). As a consequence, self-tracking has attracted a lot of attention from researchers and public opinion makers owing to its potential for improving life conditions through preemptive action on health, and as a tool of user empowerment vis-à-vis health care professionals and private and public institutions.

Nevertheless, the 'stuff' that is typically tracked - exercise and diet being the dominant tracking activities (Fox \& Duggan, 2013) - refers to cultural and social practices that, for the individual user, are utterly mundane and reside in an experiential realm of everyday life. Self-tracking activities are integrated in a 'process of confluent spheres, meanings and actions' (Pilgaard, 2012, p. 31) in which individual, acts ascribe meaning to everyday life and mediate the conditions of working life, family life and 
Lomborg, S. \& Frandsen, K. (2016). Self-tracking as communication. [pre-print version]

leisure time as a whole (Pilgaard 2012, Lomborg 2014). Seen from this perspective selftracking has to be understood in relation to behavior that is predominantly about getting things done in ways that are possible, suitable and meaningful for the individual. As we will argue - self-tracking is not just meaningful in a rational or instrumental, utilitarian sense, but also in the sense of being a source of joy and pleasure for the individual. To account for the meanings of self-tracking, we propose to conceptualize self-tracking as a social and cultural practice that is fundamentally communicative: it mirrors and molds the user (Riegeluth, 2014) towards an audience comprising to the very least the user herself, but often other users of a given service as well.

Given the diffusion and further development of self-tracking technologies it becomes crucial to elaborate our understanding of this phenomenon. Our aim in this article is to contribute to this not only by developing a certain theoretical perspective centered on communication, but also by drawing on empirical knowledge about what users actually do with self-tracking. That is, we present findings from a qualitative study on how self-tracking is practiced and experienced in the context of exercise by different categories of empirical users. We demonstrate that the meanings of self-tracking practices, on the one hand, are shaped by the motivation of an individual user who is situated in a broader web of everyday activities. On the other hand, the experiential value and meaning is not only a matter of the individual user, but also stimulated and augmented by communicative features provided by the technology. In that sense we draw upon a notion of self-tracking technologies as digital media, that possess various affordances - meaning that they have certain technological, aesthetic and social potentials, but they are defined through their actual social use and thus also formed by the needs of the users (Hutchby, 2000, 2001). 
Lomborg, S. \& Frandsen, K. (2016). Self-tracking as communication. [pre-print version]

We start by reviewing existing research on self-tracking to map the current research agenda and clarify the potential contribution of media and communication research in this context. Next, we develop a theoretical conceptualization of self-tracking as a communicative phenomenon along three dimensions addressing the relation between the user and the tracking technology, the user and the self, and the user and a wider social network of peers, respectively, Each dimension is elaborated using examples from our empirical study of exercise and self-tracking.

\section{State of the art of research on self-tracking}

In the scholarly literature, the phenomenon of self-tracking and its equivalent labels of life-logging, personal analytics, quantified self, and self-monitoring (cf. Lupton, 2014) have particularly been associated with mobile digital media that individuals carry around as they go about their daily business. Smartphone applications and 'wearables' such as the Fitbit and Jawbone Up wristbands log movements, moods, heart rates, calorie intake and so on either automatically (i.e. through sensors built into the technologies) or by individual, manual registration throughout the day. While the very practice of keeping log of specific aspects of the self and daily life goes well beyond bits and bytes and into analogue systems (pen and paper, or simply human memory) (Fox \& Duggan, 2013), digital media have certainly enabled new, easy and effortless means of self-tracking. ${ }^{1}$

1 Our focus is on digital media, but the communicative perspective we advance in principle includes all kinds of media used to keep log of the self, and often, new forms of self-tracking will have strong affinity with earlier, analogue, forms (as will be evident below). 
Lomborg, S. \& Frandsen, K. (2016). Self-tracking as communication. [pre-print version]

There are very few explicit definitions of self-tracking. Some approach selftracking from a technical perspective and define self-tracking in terms of the digital systems and devices that allow users to collect, analyze and reflect upon their data (e.g., French \& Smith, 2013; Li, Dey, \& Jodi, 2010). Others examine self-tracking as a practice of collecting, accumulating and making visible everyday habits and bodily reactions in order to reflect upon and regulate these (Ruckenstein, 2014: 68-69; also Swan, 2009; Choe et al., 2014). In this article, we follow Lupton (2014) who combines the technological and practice dimensions and defines self-tracking as the individual's use of technology to record, monitor and reflect upon features of daily life. According to Lupton, self-tracking with digital media can assume a number of forms, depending on whether or not it is voluntary (i.e. initiated by oneself) and whether it is a private or communal practice.

In the research literature on self-tracking, there are three clearly discernable bodies of literature which differ in terms of their disciplinary embedding and associated research interests. The application of self-tracking has been studied in the context of a) health care and b) interaction design and systems development research, and c) the implications of self-tracking have been discussed under a critical-sociological lens in terms of surveillance, labor and loss of privacy.

The application of self-tracking in the health care sector has been studied through interdisciplinary work on health informatics systems at the intersection of computer science and health studies. This strand of research centers on how selftracking technologies may be used by citizens to prevent and identify health issues with a view to optimizing health care services and the sector as such (Swan, 2009, 2012). A 
Lomborg, S. \& Frandsen, K. (2016). Self-tracking as communication. [pre-print version]

key idea is that self-tracking, because of its making visible patterns regarding calorie intake, exercise, sleep, and so on, may be seen as a resource for empowering the individual user vis-à-vis health care professionals. By displaying and giving feedback to users on their health in real-time and over time, self-tracking enables 'self-care' (Hansen, 2012), but it also shifts the responsibility for good health from the professional system to the individual (Lupton, 2013b). Accordingly, a number of empirical studies have investigated how self-tracking technology is used to 'nudge' users to change habits and develop a healthier lifestyle, for instance, by helping them to loose weight (TurnerMcGrievy et al., 2013; Wang et al., 2014). Other studies have examined the effects of selftracking apps on disease treatment management for citizens living with chronic diseases such as diabetes or cancer (for a review of such studies, see Wang et al., 2014). Yet other studies have examined self-tracking as a tool for improving communication between patients and health care professionals (Chiang, Yang, \& Tu, 2014; Steele, 2013).

Another strand of computer science research on self-tracking focuses specifically on the optimization and recommendations for the design of self-tracking services, typically based on studies of the user experience with concrete self-tracking apps (e.g., Ahtinen, Isomursu, Ramiah, \& Blom, 2013). The key aim is to identify factors in the design and in the user that impede and motivate a certain user experience and an associated beneficial behavior (e.g., Kim, 2014; Kranza et al., 2013). For instance, Li et al. (2010) surveyed users' experienced problems in self-tracking systems and developed a stage-based model for designing persuasive technologies. In a similar vein, Epstein, Cordeiro, Bales, Fogarty, and Munson (2014) tested the user experience of visualizations of complex self-tracking data to identify visualization methods that are intuitively 
Lomborg, S. \& Frandsen, K. (2016). Self-tracking as communication. [pre-print version]

appealing and useful for the self-tracking user to discover opportunities for behavior change (also Consolvo et al. (2008)).

The third body of research, associated with surveillance studies and other critically-informed sociological analyses, has developed in response to what is perceived as an overly optimistic view of self-tracking as empowering and relegating responsibility to the individual, and as an instrument for self-improvement through close monitoring of bodily and other signals and practices. This positive discourse, it is argued, is dominant in the health and design literatures (e.g., French, 2013), perhaps chiefly owing to their applied research aim. In contrast, critical sociological analyses of self-tracking are mainly theoretical in nature, and seek to uncover the implications of self-tracking for the users. One strong line of argument in this body of literature concerns surveillance as an implicit part of self-tracking. When we record our data in digital systems, we are not only able to monitor, analyze and optimize ourselves; we also become part of systems where our data are accumulated and analyzed by a service provider, and often sold to unidentified third-party companies. The use of user data to monetize and improve products and services and target users better has been critically examined as processes of commodification and exploitation of user labor (Klauser \& Albrechtslund, 2014; Till, 2014). Furthermore, users have very little knowledge of who gets to see and benefit from their data, and this raises issues of privacy (Patterson, 2013). Drawing on Foucault's notions of the panopticon and the resulting subjectivation, for instance, Lupton (2013a; 2013b; 2014) and Reigeluth (2014) argue that self-tracking technologies and data are far from neutral. Rather they may be seen as normative engines that produce an objectivation of human subjects as entities that are defined by, augmented by and may be controlled through their numbers (Lupton, 2013a, 2014). 
Lomborg, S. \& Frandsen, K. (2016). Self-tracking as communication. [pre-print version]

Ruckenstein (2014) dubs this conversion of the human body into numbers and visualizations 'data doubles', a concept that may be useful for exploring the relationship and communication between the self and the selftracking app.

In addition to these consolidated bodies of literature, a few studies have been published on the purpose of and motivations for self-tracking in everyday life. Choe, Lee, Lee, Pratt, and Kientz (2014) studied the motivations and practices of extreme self-trackers affiliated with the quantified self-movement ${ }^{2}$ with a specific view to barriers for self-tracking. Stragier and colleagues, rooted in communication studies, have explored users' motivations for sharing exercise on social media (Stragier \& Mechant, 2013; Stragier, Mechant, \& De Marez, 2013). These studies reflect an individual-psychological and utilitarian perspective on self-tracking akin to that of the health and interaction design perspectives. Countering this, Rooksby, Rost, Mossiron, and Chalmers (2014) in computer science did a qualitative interview study of everyday tracking stressing the role of context and affect. They identified a set of distinct styles of personal tracking that were grounded in users' life histories and social contexts, and did not primarily evolve around specific rational goals, but were deeply affective in character. These styles, in turn, represent what they label 'lived informatics'.

In sum, the existing bodies of literature suggest a significant scope and breadth in self-tracking research, but the very integration of self-tracking practices in the broader cultural and structural formation of everyday life is surprisingly absent from view - both in theoretical and empirical work on self-tracking. Apart from a few highly insightful analyses (e.g., Lupton, 2014; Reigeluth, 2014; Rooksby et al., 2014; Ruckenstein, 2014), we know very little about and have very few theoretical tools to ${ }^{2}$ http://quantifiedself.com. 
Lomborg, S. \& Frandsen, K. (2016). Self-tracking as communication. [pre-print version]

grasp what is actually going on in the user's practical engagement with concrete selftracking technologies, the data they collect, and the communicative networks and personal trajectories in which they are embedded.

We propose that a media and communication studies perspective enables an analysis of self-tracking that addresses these issues, by framing self-tracking as a meaningful, even pleasurable, experience deeply interwoven in the fabric of everyday life. A communicative understanding of self-tracking highlights meaning-making as a key aspect of the appropriation and use of self-tracking technologies. Meaning-making, in turn, is contextually embedded in dynamics of interpersonal and group affiliations that are practiced in everyday life, as well as in the ongoing practical organizing of the everyday. Hence, meaning-making of self-tracking is informed not only by the individual users' cognitive and affective capacities, but also the context of use, and the communicative affordances of the technology at hand.

\section{Method}

The empirical data that we use to develop our conceptualization of self-tracking as communication, stems from a qualitative study of self-tracking for exercise with a purposeful sample of twelve Danish respondents aged 25 to 40 . Our sample included six men and six women and both avid, experienced recreational athletes and beginners. They were all regular users of various types of fitness apps (Garmin Connect, Garmin Express, Strava, RunKeeper, Endomondo and Workout Trainer by Skimble), which provide a diverse set of affordances for exercise and social networking. Denmark is characterized by a remarkable recent equalization in relation to gender and sports but also an increase in sports and exercise activities among the 25-to-40-yeal-olds. Roughly 
Lomborg, S. \& Frandsen, K. (2016). Self-tracking as communication. [pre-print version]

speaking, the cohort of 25-to-40-year-olds is in a specific life phase with a strong focus on parenting and early career. Arguably, the structure of everyday life in this cohort makes it particularly challenging to integrate a stable participation in sport and exercise (Laub \& Pilgaard, 2013; Pilgaard, 2012). Self-tracking technology might provide a particularly attractive and relevant support structure for exercise. Owing to its communicative affordances, self-tracking technology allows for both reflexivity and a creation of flexible routines, which Pilgaard (2012) has pointed out as two paradoxical but prevalent needs in late-modern sports and exercise participation.

The study combines two sets of successive qualitative data: first a onemonth registration of the activities of each respondent logged and visualized by the apps he or she used for exercise (training patterns, communications, etc.), and then semistructured qualitative interviews with the respondents about their uses of the app and the role of exercise in the respondents' current daily lives. The interviews also documented respondents' life story of sports, as bodily memory of earlier experiences with sport inform people's ways of engaging in sport and physical exercise (Pilgaard 2012).

\section{Self-tracking as a communicative phenomenon}

In this section, we conceptualize self-tracking as a communicative phenomenon, specifying how this theoretical contribution addresses existing blind spots in the scholarly literature. Basically, we apply a ritual view on communication (Carey, 1992 (1989)), which stresses the symbolic and shared production of reality in communicative actions as equal to the transmission of information. Communication is considered a 
Lomborg, S. \& Frandsen, K. (2016). Self-tracking as communication. [pre-print version]

symbolic process, where shared beliefs are formed and maintained, and reality is reproduced and perhaps even transformed. When technologically mediated, the form and content of communication are negotiated by the communicative affordances of the medium at hand, as well as by the social norms that have been constituted around its uses (Hutchby, 2001). ${ }^{3}$ Applying a ritual view on mediated communication means that we are particularly interested in how specific acts of communication - in our case the self-tracking practices of the sampled users - construct, share and maintain certain values and beliefs in an everyday context.

Our conceptualization of self-tracking is based on two premises. Firstly, self-tracking technologies are defined as media that enable a diverse but interrelated set of activities including accumulating a tracking log or diary in a digital system, interacting with the analysis features that are often built into the interface of tracking applications, sharing tracking activities and communicating about self-tracking with others. These activities are central for our conceptualization as they are fundamentally communicative, although in very different ways, representing at once processes of conveying information about oneself to others and to a system, of constituting and performing oneself, and upholding a set of social relations in a particular way (Carey, 1992 (1989)). Secondly, for the user, the activity of self-tracking is a habitual practice of media use that is deeply interwoven in other social and cultural practices (bodily practices, daily rhythms etc.) and must thus be analyzed not in isolation, but with a view to its broader function in the users' orchestration of the demands, structures and needs of various contexts in everyday life. This ritual, 'non-media-centric' and practice oriented approach (Moores, 2012) contributes with a highly relevant perspective as it

3 This view to a large extend aligns with the tradition of Medium Theory (e.g., McLuhan, 1964; Meyrowitz, 1985), and phenomenological perspectives on media use (Scannel, 1995). 
Lomborg, S. \& Frandsen, K. (2016). Self-tracking as communication. [pre-print version]

appreciates the particularities of media technology but take its primary analytical point of departure in the communicative practices of the users and their everyday context.

In the following sections, we unpack our conceptualization of self-tracking as communication along three interrelated dimensions: as a communicative practice of interacting with and accumulating data in a digital system (i.e., a specific exercising app, but also a complementary set of networked connected media such as a digital wristband and a smartphone app); of communicating with oneself (as represented through data) and acting on the self, and of communicating with peers.

\section{Communicating with a digital system}

When we use digital media, we 'speak into systems' of communication (Jensen, 2013). We imprint our activities as digital bit trails - data that are received, analyzed, stored and often repurposed by the providers of the services we use for system refinement and personalization of future user experience with the service, and targeted advertising. That is to say, the system or medium 'responds' to the bit trail, makes sense of the data and feeds back to the user, as well as forward to others. Although we rarely think of these data in terms of communication, perhaps we should. Unfolding a theoretical analysis of digital data as communication and meta-communication, Jensen (2013) argues that, just as words 'do' things (Austin, 1962), digital data are performative - at once vehicles of information and sources of meaning that construct and act on reality in myriad ways. When I log onto Endomondo and track a run, the information in data points collected on my pace, geo-location, etc. are processed by and contribute to finetuning Endomondo's algorithms and thus, however minimally, reconfigure the system. It 
Lomborg, S. \& Frandsen, K. (2016). Self-tracking as communication. [pre-print version]

may also lead to renewals and expansion in the whole system, like when Endomondo starts e-mailing me on a monthly basis summing up my exercise in acclaiming terms, and urging me to exercise even more, keep up the motivation by strengthening the social network on the service and using the support functions provided. Similarly, the metadata that accompany communications with peers in the system about the exercise or customize their profile (time-stamps, details on the platform used, etc.) not only codify the communication and relationship between users, but also meta-communicate meaning about the user's sports identity. In our data this is distinctly manifested in the users' choice of photo. Their photos convey meaning about their type of engagement in exercise, which is strategically and commercially valuable for the provider of the system. Among those users that have a long trajectory in sports and exercise, the photo is used in a very self-reflexive and context-sensitive manner. The users distinguish and display this part of their identity with photos showing themselves in sportswear, among other athletes or otherwise referring to their training practices. They communicate a sports identity and thus suggest a particular kind of social relationship - centered on sports that they wish to express on the app. The respondents for whom sport and exercise has not had a high priority in earlier stages of life use other kinds of profile photos, if any at all. Typically, they simply use their Facebook profile photo, which can be fetched automatically through systems such as Endomondo, thus conveying no intent of marking their use of self-tracking as reflecting a strong sports identity.

Our user's responses to the 'system's communication' take affective and normative forms expressed in terms of annoyance, irritation and pleasure. For Alice 
Lomborg, S. \& Frandsen, K. (2016). Self-tracking as communication. [pre-print version]

$(40)^{4}$, a beginner in physical exercise but a professional in information technology and avid user of apps, it was a great source of irritation that her preferred app (that was used by a lot of people in her offline social network) did not allow for integration with other lifestyle apps. She laments this as blatant self-sufficiency and inappropriate lack of openness in the technology. The student Tommy (26), who is a well-experienced athlete with a strong fascination of technological sports equipment, responds to the same app's use of burgers as icons for burned calories with this statement: 'This is the amount of burgers that you have burned (...) I don't care (...) This is a load of crap.' For him the system communicates cultural values that he considers totally inappropriate for his performance of an identity as a serious, competitively oriented athlete. He and other dedicated athletes in the sample, such as Martin (36) and Sandra (34) mainly appreciate systems features such rankings, detailed information about training sessions, and flexibility in planning tools, as these features communicate various values associated with serious sports.

Self-tracking systems are also communicative in a more explicit manner. Some apps afford instant accompanying verbal feedback during a training session. Although feedback may come from the social network, our respondents did not use this feature. One exception is the schoolteacher Peter (31), who sometimes is 'pep talked' by his Endomondo friends, and appreciates this feature to the extent that he sometimes changes his use of technology in order to benefit from this facility: 'it is more comfortable to run with the watch than having a big telephone on your arm (...) But sometimes it is fine to get a peptalk , and that is not possible with the watch...'.

\footnotetext{
${ }^{4}$ All participants are pseudonymized, whereas their real age is disclosed.
} 
Lomborg, S. \& Frandsen, K. (2016). Self-tracking as communication. [pre-print version]

Most often it is the system itself that communicates - personified as a speaking voice stating time laps and distance passed in training. Alice (40) perceived this feature as an omni-present personal coach that informs her about progress and gives other kinds of feedback in the course of and evaluating a training session. This kind of personified feedback provides a structure that for the respondents is meaningful in the situation. But it also becomes meaningful in a wider sense. The accumulation of information that is archived and communicated back to the users makes up an explicit individualized performance history that is considered particularly helpful by some users. For Carol (27) and Eric (34), who both have small children and no fixed timeslots for exercise, this functions to keep them on track and as 'a reminder to myself, that I should not spend money on this without using it. So, in this way it ignites me. Last month was not optimal, tighten up!' (Eric, 34). For these two users the communication back from the system becomes a very meaningful resource to negotiate the structure of their everyday life and secure time for sport and exercise.

For Martin (36) and Kenneth (30) the systems' communicative feedback is so important that it is extended and gets personalized as they combine several technologies in their training practice in order to get feedback from a wider set of digital sources. Kenneth connects the Strava app on his mobile phone with a Garmin watch via Bluetooth. Besides giving him various kinds of feedback this setup also makes it possible for his family to follow him simultaneously on their computer screen. For Martin the objective of using both a Garmin watch and Endomondo's app meets a complex need for detail in feedback in detail while also maintaining an overview: 'Endomondo is just running on its own. And then my watch - I'm using it actively to set my pace (...) Endomondo, I use it mostly to look for my split times and things like that.' Both Martin 
Lomborg, S. \& Frandsen, K. (2016). Self-tracking as communication. [pre-print version]

and Kenneth thus seek to optimize their practice by combining response from several systems, thus creating a whole new structure accommodating these systems of communication to their own personal needs.

\section{Communicating with and acting on the self}

A core dimension of self-tracking with digital applications are data visualizations of the accumulated input the user has offered the system: ranging from general plots of exercise activity over an extended period, to more fine-grained visualizations of, for instance, routes or the users' pulse during one exercise session. These visualizations clearly communicate: they provide feedback to the user on stability and changes in training patterns, bodily strength and so on. In turn, they function as a mirror for the self, a means of communicating with the self ('how well did I do today compared with the past sessions, and what does that say about my shape?').

In the context of self-tracking, Ruckenstein (2014) has developed the concept of the 'data double', originally conceived in surveillance studies, to describe the auto-communicative relationship between the user and the data visualization of her tracking activities. ${ }^{5}$ The data double denotes 'the conversion of human bodies and minds into data flows that can be figuratively reassembled for the purposes of personal reflection and interaction' (Ruckenstein, 2014: 68). Hence, the data double turns hitherto invisible bodily and mental details into coherent pieces of information that the user can engage and interact with in order to better understand the self. According to Ruckenstein, who empirically studied the use of heart rate monitoring in everyday life,

\footnotetext{
5 The concept of auto-communication, originally developed in cultural semiotics by Lotman (1990), suggests that all communication implies a relationship between the communicator and herself.
} 
Lomborg, S. \& Frandsen, K. (2016). Self-tracking as communication. [pre-print version]

the relationship between the user and her data double is affective and purposeful something that is often understated in the surveillance literature. Rooksby et al. (2014) also point to the affective dimension and they relate it to an experience of self-esteem. We would argue that the pleasurable feeling that our users associate with self-tracking is very much rooted in the data visualizations that the systems provide. Firstly, because the visualizations provide them with a tool for short-term or long-term reflection on their bodily practices, and thus prolong and augment the exercise session - as a psychological experience. Secondly, the rhetorical form of visualizations is important in itself: charts, tables, icons and factual data seem to associate tracking with science and accordingly also with values like seriousness, analysis and competent expertise. Arguably, this supports the gratification of basic psychological needs that communication scholars have elsewhere argued motivates the use of entertainment products (Vorderer, Steen, \& Chan, 2006). According to Vorderer and colleagues (2006) people often use media to meet three basic psychological needs: 1) A need to be autonomous and in control, 2) a need to feel competent, and 3) a need to feel related to others. Following this line of theory the positive affective response to self-tracking reflects the gratification of individual users' needs - because the visual data establishes a communicative seriousness and respect around the individual, no matter his or her level of exercise.

The nature of our users' engagement in and interaction with the visualizations varies a lot. As Rooksby et al. (2014) have noticed, most users - including those we studied - deal with these data on a short term basis. Most of the users report a particular focus on the data, like Eric (34) on burnt calories, Alice (40) on time spent and length of a run, and Helen (36) on pulse. Most of them engage with these data via their 
Lomborg, S. \& Frandsen, K. (2016). Self-tracking as communication. [pre-print version]

mobile phone for a short while immediately after finishing the exercise session. They seek immediate feedback on certain aspects. To understand the full meaning of this communication we need to take into account the cultural meaning of the 'scientific' mode of address and the meaning of the communicative action itself. A user who only spends a little more than a minute looking at her route and her average pace is confirmed through the data double that she is as a respectable individual who is competently taking agency in her own life. The act of looking at these visualizations are not only serving as an evaluation, it may also be considered a communicative ritual as it constitutes a moment when the exercise experience is prolonged, while at the same time changed from a bodily experience into a psychological experience where documentation allows for gratification. The experiential mode of 'sensing' the body is transformed into a communicative mode where 'seeing' a certain kind of representation of the very same session adds new meaning. This transformation into the communicative mode of 'seeing' appears particularly important for beginners like Alice (40), who explicitly says that she feels less capable in sensing her own body. For experienced athletes like Terry (37) and Helen (36) the doubling of the exercise experience constitutes an attractive and joyful experiential space of auto-communication. In this space they come to know themselves as in control and very competent, systematically oscillating between modes of 'sensing' and 'seeing' in a very reflexive search for improvement of their own performance.

The system's accumulation of information basically assures all users that they keep track of their own life - and thus are in control. But a few, like Kenneth (30) and Tommy (26) and spend more time on exploring the data, and this interaction takes place at a later hour, where they have plenty of time for themselves. For these two users sport and exercise has been a constant and important element throughout their life, and 
Lomborg, S. \& Frandsen, K. (2016). Self-tracking as communication. [pre-print version]

they express great joy when indulging themselves with the data in search for both personal advancement and competitively oriented comparisons with other users.

\section{Communicating with peers}

Self-tracking technologies such as Strava, Endomondo, Garmin Connect and Runkeeper allow the user to establish connections and communicate with other users through the technology. In that sense, self-tracking embeds key functionalities from social media, for networking, sharing, liking, and commenting that are further shaped by the users' communicative practices onsite. Following from this perspective, studying self-tracking as a communicative practice has close affinity with research on computer-mediated communication (CMC) as a vehicle of sociality (e.g., Bakardjieva, 2005; Baym, 2000; Kendall, 2002). This extensive body of literature has, for instance, demonstrated how people interlace and develop relationships across contexts on and off digital media in everyday life (e.g., boyd, 2008; Ellison, Steinfield, \& Lampe, 2007; Lomborg, 2014; Wellman \& Haythornthwaite, 2002).

Under the lens of sociality, communication is predominantly a means of achieving a common practice and shared understanding of the situation at hand, of maintaining social order and thus finding meaning and pleasure in a sense of belonging (Carey, 1992 (1989); Vorderer et al., 2006). That is to say, the practice of communicating with others implies an ongoing negotiation of what is appropriate, relevant and expected to be communicated in a given context and with a given set of people.

In our data, the degree of connectedness and the amount of communication with one's network on self-tracking services varies a great deal. Strikingly, 
Lomborg, S. \& Frandsen, K. (2016). Self-tracking as communication. [pre-print version]

communication on self-tracking services is most prolific for the participants who practice their sports with others, and who use the togetherness and competition in the joint practice of exercising as a motivator. In the registered data from their self-tracking profiles, communication typically takes the form of descriptions and evaluations of individual training passes, which may then receive likes and supportive comments. This indicates a strong topical focus on exercise as appropriate for the context at hand. It also highlights social recognition of an individual's efforts as an important part of the selftracking experience for some of the users. The activity thus becomes socially meaningful as it meets a need to feel communion with others (Vorderer et. al. 2006). Here we find parallels to dynamics that are well known and central in social media in general (Lomborg 2014) but also to mechanisms that constitute sports culture, where social recognition from a knowledgeable audience is an important tool when celebrating a winner (Frandsen, 2013). Sandra (34), who has found communion with work colleagues in running and tracking it on Endomondo, asserts about self-tracking: 'it is all about supporting one another in the place that we are each in, and "it is so cool that you do this" and the like'. Crucially, the recognition and support is sought from the 'relevant others'. In the case of the users communicating on Endomondo, Strava, etc., the relevant others are those with whom the individual user shares the exercising as a common topic and activity in everyday life. Peter (31) and Sandra (34) both use exercise as a way of socializing with colleagues through small talk at work, on Endomondo, and sometimes through running together. Hannah (39), who is a member of a serious running club and whose everyday social life evolves around running, receives many likes and has lively conversations about her exercise passes on Endomondo with her running mates, after each completed session. Accordingly, the communication on the self-tracking services 
Lomborg, S. \& Frandsen, K. (2016). Self-tracking as communication. [pre-print version]

thereby extends in time, and perhaps augments, the social experience of training with others.

To be sure, communication related to self-tracking of exercise not only occurs on the self-tracking applications. Kenneth (30), who uses Strava and keeps a very small and close network of four close friends with whom he cycles reveals having a lot of ping-pong about cycling through email and in face-to-face communication with his Strava-friends. Terry (37) has started a Facebook-group where he and his peers share training data and support one-another. For these users, concrete instances of tracking, shared with peers on the self-tracking applications, serve as a conversation-starter in other contexts.

Other participants, particularly a subset of the users who only exercise alone, do not communicate about their exercise on their preferred self-tracking service, but some of them post auto-updates on their exercise activities to Facebook, thereby sharing their exercise with a wider network of friends. Carol (27) and Doris (29) both use sharing on Facebook as part of their individual exercising projects. Creating awareness in the social networks about their goals and practices of exercising seems to inspire them to stay on track. For instance, Doris, who is a newbie to exercising in general and is in the process of losing weight after a pregnancy, describes her automatic sharing of exercise on Facebook as simply a way of letting people know what she is occupied with - perhaps as a way of motivating herself to continue when receiving supportive comments and likes from her Facebook friends. The culture of recognition that we have identified on self-tracking services as well as in other contexts is well documented as a crucial function of CMC in general, for instance in the form of phatic communication (Lomborg, 2014; Miller, 2008). Communicating about exercise, and 
Lomborg, S. \& Frandsen, K. (2016). Self-tracking as communication. [pre-print version]

specifically the sharing of training results with others has implications for and feeds back into the training practices of the self-trackers: most of them says it means that they pull themselves together and give their exercise an extra nudge because it is on display for others. For some of the participants, communicating about exercise on Facebook seems to serve a function beyond motivating oneself, namely, to urge others to exercise as well. Alice (40) describes her sharing on Facebook as intended by a wish to motivate others: 'when people know me as someone who never exercised at all and who was very overweight, then if I can run five kilometers, then everybody can run five kilometers'.

At the same time, several participants also voice a concern with sharing exercise with broader social networks such as on Facebook. The concern has to do with a perceived conflict with broader social norms of what is relevant for the context. As Eric (34) contends: 'I get really annoyed with my own Facebook friends when they post all this "I've been out running four kilometers". Well, that is super for you. But I like it in the application, because to me it belongs in that universe, and the peers who are there are there with that specific purpose'. Strikingly, particularly the men in the sample voice this idea of confining exercise communication to specific relevant contexts dedicated to specific purposes. For some of the women, in particular, the sharing of tracking as such is ambivalent. Carol (27) worries that by letting others see how much exercise she fits into her everyday schedule will communicate an unwanted idea of her as a bad mother who does not give priority to being with her kids. Similarly, Helen (36) worries about being labeled as obsessed with training. What these women seem to suggest is that exercising and sharing it is weighed against other social norms and expectations of normality (e.g., not being fanatic about training so that other needs and activities are compromised). 
Lomborg, S. \& Frandsen, K. (2016). Self-tracking as communication. [pre-print version]

In terms of their communicative functionalities for sharing individual exercise, self-tracking services may be said to fit with what Castells has dubbed 'mass self-communication' (Castells, 2007), that is, individualized and self-centered communicative statements distributed online to a potential mass audience. However, the communicative practices of the users in this study tell a different story. Overall, their onsite communication is fairly limited, and so are the social networks on the selftracking applications of most of the participants, who orient themselves towards a small group of relevant others. This is surprising, given that a key experiential offer of the applications is to add an element of sociality to the often-individual exercising habits (running, cycling, etc.). Moreover, rather than self-tracking fundamentally altering the exercising practice, what we have seen is that the practices of exercising alone or with others reflects in the use of and communication on the self-tracking application. Those who exercise together tend to communicate and socialize more in the self-tracking system. Hence, when social networks matter on the tracking services, it is because these networks are kept vibrant in other contexts in everyday life. Self-tracking technologies may thus assist in maintaining and amplifying existing relationships.

\section{Conclusion: Self-tracking in an everyday context}

We have developed a communicative perspective on self-tracking by examining the use of self-tracking in the context of exercise in everyday life along three dimensions: communication with the system, the self and social networks. The communicative affordances of self-tracking applications provide a flexible and social structure for exercising, one that participants come to see as pleasurable and meaningful, in great 
Lomborg, S. \& Frandsen, K. (2016). Self-tracking as communication. [pre-print version]

part because it is scalable to their individual and social needs. The structures and demands of everyday life, and the social relationships participants maintain in diverse contexts, manifest themselves in each of the communicative dimensions examined: the system provides an infrastructure for planning and executing exercise in a personalized manner. That is to say, the system is responsive to individual user needs. The feedback provided by the system on exercising metrics, both on individual exercising sessions and accumulated patterns over time, functions as a basis for adjusting training goals, for instance by prompting the user to increase the amount of exercise. And the opportunity to network with relevant others on the applications serves to integrate exercise in other everyday contexts (i.e., work, leisure, family life). Approaching self-tracking from the perspective of communication theory makes visible the relationship between selftracking practices and their contextual embedding: what is communicated on the app (in the form of profile information, exercise data and social networks) bears traces of the contexts of training. Moreover, this communication serves to constitute the users' sense of self in the context of exercise: profile information, data visualizations and feedback from the system and the connected peers contribute to confirming the users' exercise identity, competence and agency. Arguably, this is a crucial part of what makes selftracking pleasurable, motivating and meaningful for users.

\section{References}

Ahtinen, A., Isomursu, M., Ramiah, S., \& Blom, J. (2013). Advise, Acknowledge, Grow and Engage: Design Principles for a Mobile Wellness Application to Support Physical 
Lomborg, S. \& Frandsen, K. (2016). Self-tracking as communication. [pre-print version]

Activity. International Journal of Mobile Human Computer Interaction (IJMHCI), $5(4)$.

Austin, J. L. (1962). How to do things with words. Oxford: Clarendon Press.

Bakardjieva, M. (2005). Internet society. The Internet in everyday life. Thousand Oaks, CA: Sage Publications.

Baym, N. K. (2000). Tune in, log on. Soaps, fandom, and online community. Thousand Oaks, CA: Sage Publications.

boyd, d. (2008). Taken out of context. American teen sociality in networked publics. (PhD), University of California, Berkeley.

Carey, J. W. (1992 (1989)). Communication as culture. Essays on media and society (Reprinted ed.). New York London: Routledge.

Castells, M. (2007). Communication, Power and Counter-power in the Network Society. International Journal of Communication, 1, 238-266.

Chiang, J.-H., Yang, P.-C., \& Tu, H. (2014). Pattern analysis in daily physical activity data for personal health management. Pervasive and Mobile Computing, 13, 13-25.

Choe, E. K., Lee, N. B., Lee, B., Pratt, W., \& Kientz, J. A. (2014). Understanding quantifiedselfers' practices in collecting and exploring personal data. Paper presented at the CHI 2014, Toronto, ON.

Consolvo, S., Klasnja, P., McDonald, D. W., Avrahami, D., Froehlich, J., LeGrand, L., . . . Landay, J. A. (2008). Flowers or a Robot Army? Encouraging Awareness \& Activity with Personal, Mobile Displays. Paper presented at the UbiComp '08 Proceedings of the 10th international conference on Ubiquitous computing. 
Lomborg, S. \& Frandsen, K. (2016). Self-tracking as communication. [pre-print version]

Ellison, N. B., Steinfield, C., \& Lampe, C. (2007). The benefits of Facebook 'friends': Social capital and college students' use of online social network sites. Journal of Computer-Mediated Communication, 12(4), 1143-1168.

Epstein, D. A., Cordeiro, F., Bales, E., Fogarty, J., \& Munson, S. A. (2014). Taming Data Complexity in Lifelogs: Exploring Visual Cuts of Personal Informatics Data. Paper presented at the DIS '14 Proceedings of the 2014 conference on Designing interactive systems.

Fox, S., \& Duggan, M. (2013). Tracking for health: Pew Internet \& American Life Project. Frandsen, K. (2013). In a different game?Reflections on sports in the media as seen from a game perspective. In P. M. Pedersen (Ed.), Routledge Handbook of Sport Communication. London \& New York: Routledge.

French, M., \& Smith, G. (2013). 'Health' surveillance: new modes of monitoring bodies, populations, and polities. Critical Public Health, 23(4), 383-392.

Hansen, M. M. (2012). Self-Tracking, Social Media and Personal Health Records for Patient Empowered Self-Care Nursing and Health Professions Faculty Research. Paper 17.

Hutchby, I. (2000). Conversation and technology: The telephone, the computer, and the nature of human communication. Cambridge, UK, Malden, MA.: Polity Press.

Hutchby, I. (2001). Technologies, Texts and Affordances. Sociology, 35(2), 441-456.

Jensen, K. B. (2013). How to do things with data: meta-data, meta-media, and metacommunication. First Monday, 18(10).

Kendall, L. (2002). Hanging out in the virtual pub: Masculinities and relationships online. Berkeley, CA: University of California Press. 
Lomborg, S. \& Frandsen, K. (2016). Self-tracking as communication. [pre-print version]

Kim, J. (2014). Analysis of health consumers' behavior using self-tracker for activity, sleep, and diet. Telemedicine Journal and E-Health, 20(6), 552-558.

Klauser, F. R., \& Albrechtslund, A. (2014). From self-tracking to smart urban infrastructures: towards an interdisciplinary research agenda on Big Data. Surveillance \& Society, 12(2), 273-286.

Kranza, M., Möller, A., Hammerlac, N., Diewald, S., Plötzc, T., Olivierc, P., \& Roalter, L. (2013). The mobile fitness coach: Towards individualized skill assessment using personalized mobile devices. Pervasive and Mobile Computing, 9(2), 203-215.

Laub, T. B., \& Pilgaard, M. H. (2013). Danskernes motions og sportsvaner 2011. Copenhagen: Idrættens Analyseinstitut (IDAN).

Li, I., Dey, A., \& Jodi, F. (2010). A Stage-Based Model of Personal Informatics Systems. Paper presented at the CHI '10, Atlanta, Georgia.

Lomborg, S. (2014). Social media - social genres. Making sense of the ordinary. London and New York: Routledge.

Lotman, Y. (1990). Universe of the mind. A semiotic theory of culture. Bloomington and Indianapolis: Indiana University Press.

Lupton, D. (2013a). The Digital Cyborg Assemblage: Haraway's Cyborg THeory and the New Digital Health Technologies. In F. Collyer (Ed.), The Handbook of Social Theory for the Sociology of Health and Medicine. Houndmills: Palgrave Macmillan.

Lupton, D. (2013b). The digitally-engaged patient: self-monitoring and self-care in the digital health era. Social Theory and Health, 11(3), 257-270.

Lupton, D. (2014). Self-tracking Modes: Reflexive Self-Monitoring and Data Practices. Paper presented at the Imminent Citizenships: Personhood and Identity Politics in the Informatic Age workshop, Canberra. 
Lomborg, S. \& Frandsen, K. (2016). Self-tracking as communication. [pre-print version]

McLuhan, M. (1964). Understanding media: the extensions of man. New York: McGraw Hill.

Meyrowitz, J. (1985). No Sense of Place: The Impact of Electronic Media on Social Behavior. Oxford: Oxford University Press.

Miller, V. (2008). New media networking and phatic culture. Convergence, 14(4), 387400.

Moores, S. (2012). Media, place \& mobility. Houndmills, Basingstoke: Palgrave Macmillan.

Patterson, H. (2013). Contextual Expectations of Privacy in Self-Generated Health Information Flows. Paper presented at the TPRC 41: The 41st Research Conference on Communication, Information and Internet Policy. http://ssrn.com/abstract $=2242144$

Pilgaard, M. H. (2012). Flexible sports participation in late-modern everyday life. (PhD), University of Southern Denmark. Retrieved from http://www.idan.dk/vidensbank/forskning-og-analyser?s=pilgaard

Reigeluth, T. (2014). Why data is not enough: Digital traces as control of self and selfcontrol. Surveillance \& Society, 12(2), 243-254.

Riegeluth, T. (2014). Why data is not enough: Digital traces as control of self and selfcontrol. Surveillance \& Society, 12(2), 243-354.

Rooksby, j., Rost, M., Mossiron, A., \& Chalmers, M. (2014). Personal tracking as lived informatics. Paper presented at the CHI 2014, Toronto, ON.

Ruckenstein, M. (2014). Visualized and Interacted Life: Personal Analytics and Engagements with Data Doubles Societies, 4, 68-84.

Scannel, P. (1995). For a phenomenology of Radio and Television. Journal of Communication, 45(3), 4-19. 
Lomborg, S. \& Frandsen, K. (2016). Self-tracking as communication. [pre-print version]

Steele, R. (2013). Utilizing Social Media, Mobile Devices and Sensors for Consumer Health Communication: A Framework for Categorizing Emerging Technologies and Techniques. In S. C. Mukhopadhyay \& O. A. Postolache (Eds.), Pervasive and Mobile Sensing and Computing for Healthcare (Vol. 2, pp. 233-249). Berlin \& Heidelberg: Springer

Stragier, J., \& Mechant, P. (2013, 7.-8. February 2013). Mobile fitness apps for promoting physical activity on Twitter: the \#RunKeeper case. Paper presented at the Etmaal van de communicatiewetenschappen, Rotterdam.

Stragier, J., Mechant, P., \& De Marez, L. (2013). Studying physical activity using social media: an analysis of the added value of RunKeeper tweets. International Journal of Interactive Communication Systems and Technologies, 3(2), 16-28.

Swan, M. (2009). Emerging Patient-Driven Health Care Models: An Examination of Health Social Networks, Consumer Personalized Medicine and Quantified SelfTracking. International Journal of Environmental Research and Public Health, 6, $492-525$.

Swan, M. (2012). Health 2050: The Realization of Personalized Medicine through Crowdsourcing, the Quantified Self, and the Participatory Biocitizen. Journal of Personalized Medicine, 2(3), 93-118.

Till, C. (2014). Exercise as Labour: Quantified Self and the Transformation of Exercise into Labour. Societies, 4, 446-462.

Turner-McGrievy, G. M., Beets, M. W., Moore, J. B., Kaczynski, A. T., Barr-Anderson, D. J., \& Tate, D. F. (2013). Comparison of traditional versus mobile app self-monitoring of physical activity and dietary intake among overweight adults participating in an 
Lomborg, S. \& Frandsen, K. (2016). Self-tracking as communication. [pre-print version] mHealth weight loss program. Journal of the American Medical Informatics Association, 20, 513-518.

Vorderer, P., Steen, F. F., \& Chan, E. (2006). Motivation. In J. Bryant \& P. Vorderer (Eds.), Psychology of Entertainment. London: Lawrence Erlbaum Associates Publishers.

Wang, J., Wang, Y., Wei, C., Yao, N., Yuan, A., Shan, Y., \& Yuan, C. (2014). Smartphone Interventions for Long-Term Health Management of Chronic Diseases: An Integrative Review. Telemedicine Journal and E-Health, 20(6), 570-583.

Wellman, B., \& Haythornthwaite, C. (Eds.). (2002). The internet in everyday life. Oxford: Wiley-Blackwell.

Wolf, G. (2010, 28. April). The Data-Driven Life. New York Times. Retrieved from http://www.nytimes.com/2010/05/02/magazine/02self-measurementt.html?pagewanted=all\&_r=0 